\title{
Improved SBS limited parametric conversion by use of few mode fibers
}

\author{
Mariam Mathew, Neethu; Christensen, Erik Nicolai; Grüner-Nielsen, Lars; Rottwitt, Karsten
}

Published in:

Proceedings of 2018 European Conference on Optical Communication

Link to article, DOI:

10.1109/ECOC.2018.8535345

Publication date:

2018

Document Version

Publisher's PDF, also known as Version of record

Link back to DTU Orbit

Citation (APA):

Mariam Mathew, N., Christensen, E. N., Grüner-Nielsen, L., \& Rottwitt, K. (2018). Improved SBS limited parametric conversion by use of few mode fibers. In Proceedings of 2018 European Conference on Optical Communication IEEE. https://doi.org/10.1109/ECOC.2018.8535345

\section{General rights}

Copyright and moral rights for the publications made accessible in the public portal are retained by the authors and/or other copyright owners and it is a condition of accessing publications that users recognise and abide by the legal requirements associated with these rights.

- Users may download and print one copy of any publication from the public portal for the purpose of private study or research.

- You may not further distribute the material or use it for any profit-making activity or commercial gain

- You may freely distribute the URL identifying the publication in the public portal 


\title{
Improved SBS limited parametric conversion by use of few-mode fibers
}

\author{
Neethu M. Mathew ${ }^{(1)}$, Erik N. Christensen ${ }^{(1)}$, Lars Grüner-Nielsen ${ }^{(2)}$, and Karsten Rottwitt( ${ }^{(1)}$
}

(1) DTU Fotonik,Ørsteds Plads, building 3432800 Kgs. Lyngby, Denmark namama@fotonik.dtu.dk

(2) Danish Optical Fiber Innovation, Åvendingen 22A, 2700 Brønshøj, Denmark

\begin{abstract}
We model and measure the SBS threshold in few-mode fibers, and demonstrate parametric amplification. Furthermore, we present an optimized fiber design that has a SBS limited nonlinear phase shift, higher than that of a conventional highly nonlinear fiber.
\end{abstract}

\section{Introduction}

Stimulated Brillouin scattering (SBS) is in many cases a limiting factor for nonlinear signal processing in optical fibers as it set the upper limit for the power, which can be launched into the fiber, and thereby set the maximum nonlinear phase shift. This is described through the nonlinear figure of merit $\left(\mathrm{FOM}_{\mathrm{NL}}\right)$ for SBS limited highly nonlinear fibers ${ }^{1,2}$ :

$$
F O M_{\mathrm{NL}}=\gamma L_{\mathrm{eff}} P_{\mathrm{th}},
$$

where $\gamma$ is the Kerr nonlinear coefficient, $\mathrm{L}_{\text {eff }}$ the effective length and $\mathrm{P}_{\text {th }}$ the SBS threshold. In this work we use a higher order mode in a few mode fiber as a way to increase the $\mathrm{FOM}_{\mathrm{NL}}$. Measurement and modeling results for SBS threshold are shown for two fibers supporting $\mathrm{LP}_{01}$ and $\mathrm{LP}_{11}$. For the fiber with the highest $\mathrm{FOM}_{\mathrm{NL}}$, intra modal four wave mixing results are presented as well. The examined fibers are not ideal for nonlinear signal processing as they are designed as transmission fibers. A theoretical design for an optimized two LP mode highly nonlinear fiber is presented and shown to have a high $\mathrm{FOM}_{\mathrm{NL}}$ for the $\mathrm{LP}_{11}$ mode.

\section{Modeling}

To calculate the SBS threshold from the optical refractive index profile of the fiber, the models de-

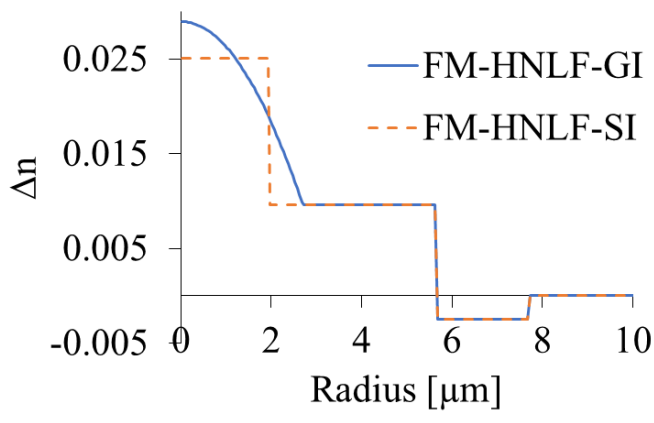

Fig. 1: Index profiles FM-HNLF scribed in ${ }^{3,4}$, have been used. From the optical refractive index profile, the radial acoustic velocity profile is found. The acoustic wave equation is then solved for guided acoustic modes. For each acoustic mode, the Brillouin frequency shift $\nu_{\mathrm{B}}$, the Brillouin bandwidth $\nu_{\mathrm{Bbw}}$, and the peak Brillouin gain efficiency $G_{0}$ are found. The later from the overlap with the optical mode ${ }^{4}$. The SBS threshold power is determined by the acoustic mode with the highest Brillouin gain efficiency $\mathrm{G}_{0 . \text { max }}$. The SBS threshold is then calculated from

$$
P_{\mathrm{th}}=\frac{q}{G_{0, \max } L_{\mathrm{eff}}},
$$

where $\mathrm{q}$ is a constant, depending on $\nu_{\mathrm{B}}, \nu_{\mathrm{Bbw}}$, $\mathrm{G}_{0, \max }$, fiber attenuation, length and finally the ratio of input power and back reflected Stokes power at threshold. In our modeling this ratio is set to 0.16 as this gives best agreement with measured threshold. $q$ is then calculated from formula (20), (27), and (28) in ${ }^{3}$.

Four fibers have been modeled. The two first are commercial fibers from OFS, a step index fiber (2MSIF) supporting two LP modes and a graded index fiber (2MGIF) also supporting two LP modes ${ }^{5}$. Both originally designed as transmission fibers. These fibers have also been used for the experimental work. However, as shown later these fibers are not ideal for use for parametric amplification as their high chromatic dispersion ${ }^{5}$ give poor phase matching. To our knowledge no few mode fibers have been realized with better phase matching. However, some theoretical designs have been proposed ${ }^{6,7}$. These fibers are designed for a zero dispersion wavelength around $1550 \mathrm{~nm}$ for both $\mathrm{LP}_{01}$ and $L \mathrm{P}_{11}$ and a high Kerr nonlinear coefficient for both modes. The third design modeled is a round version of the elliptical design proposed by Guo and co-workers ${ }^{7}$ (FMHNLF-SI). Finally, the fourth design is a proposed improved version of the Guo design (FM-HNLF- 
Tab. 1: Modeling results at $1550 \mathrm{~nm}$ for a fiber length of $1 \mathrm{~km}$.

\begin{tabular}{|l|l|c|c|c|c|c|c|c|c|}
\hline fiber type & & \multicolumn{2}{|c|}{ 2MSIF } & \multicolumn{2}{c|}{ 2MGIF } & \multicolumn{2}{c|}{ FM-HNLF-SI } & \multicolumn{2}{|c|}{ FM-HNLF-GI } \\
\hline & & $\mathrm{LP}_{01}$ & $\mathrm{LP}_{11}$ & $\mathrm{LP}_{01}$ & $\mathrm{LP}_{11}$ & $\mathrm{LP}_{01}$ & $\mathrm{LP}_{11}$ & $\mathrm{LP}_{01}$ & $\mathrm{LP}_{11}$ \\
\hline $\mathrm{n}_{2}$ & $\mathrm{~m}^{2} / \mathrm{W} \cdot 10^{-20}$ & 2.25 & 2.25 & 2.30 & 2.30 & 2.80 & 2.60 & 2.80 & 2.60 \\
\hline $\mathrm{A}_{\text {eff }}$ & $\mu \mathrm{m}^{2}$ & 215 & 210 & 96 & 128 & 24 & 68 & 23 & 63 \\
\hline$\gamma$ & $(\mathrm{W} \cdot \mathrm{km})^{-1}$ & 0.42 & 0.43 & 1.0 & 0.73 & 4.7 & 1.6 & 4.9 & 1.7 \\
\hline Attenuation & $\mathrm{dB} / \mathrm{km}$ & 0.2 & 0.2 & 0.2 & 0.2 & 0.4 & 0.35 & 0.35 & 0.30 \\
\hline $\mathrm{G}_{0, \max }$ & $(\mathrm{W} \cdot \mathrm{m})^{-1}$ & 0.070 & 0.051 & 0.144 & 0.037 & 0.745 & 0.128 & 0.609 & 0.077 \\
\hline $\mathrm{q}$ & & 17.5 & 17.8 & 16.7 & 18.2 & 14.1 & 16.2 & 14.4 & 16.9 \\
\hline $\mathrm{P}_{\text {th }}$ & $\mathrm{W}$ & 0.26 & 0.36 & 0.12 & 0.50 & 0.020 & 0.13 & 0.025 & 0.23 \\
\hline FOM $_{\text {NL }}$ & & $\mathbf{0 . 1 1}$ & $\mathbf{0 . 1 5}$ & $\mathbf{0 . 1 1}$ & $\mathbf{0 . 3 6}$ & $\mathbf{0 . 0 9}$ & $\mathbf{0 . 2 0}$ & $\mathbf{0 . 1 2}$ & $\mathbf{0 . 3 7}$ \\
\hline
\end{tabular}

Gl) where the step index core is replaced by a graded index core while still keeping the same nonlinear and dispersion properties for $\mathrm{LP}_{01}$ and $\mathrm{LP}_{11}$. The refractive index profile for the two fibers are shown in figure 1. The modeling results are summarized in table 1. Both the nonlinear refractive index $\left(\mathrm{n}_{2}\right)$ and attenuation are known to depend on $\mathrm{GeO}_{2}$ doping levels and mode overlap with the doped area. For the theoretical designs they are roughly estimated by comparing with other similar fibers. For all fibers, it is observed that the $\mathrm{FOM}_{\mathrm{NL}}$ is higher for $\mathrm{LP}_{11}$ than for $L P_{01}$. I.e. use of $L P_{11}$ for $S B S$ limited signal processing seems advantageous. However, the improvement from $L P_{01}$ to $L P_{11}$ is seen to vary depending on the fiber design. The $2 \mathrm{MSIF}$ only show a $40 \%$ improvement compared to $230 \%$ for the 2MGIF. For the FM-HNLF the FOM $\mathrm{NL}_{\mathrm{L}}$ is increased for the $\mathrm{LP}_{01}$ with $30 \%$ and for the $\mathrm{LP}_{11}$ with $80 \%$ by replacing the step index core with a graded index core. For comparison conventional single mode HNLF with a $\mathrm{GeO}_{2}$ doped core have a FOM $\mathrm{NL}$ of around $0.21^{2}$.

\section{SBS Measurements}

The Brillouin scattering measurements are done on a $10 \mathrm{~km}$ long $2 \mathrm{MSIF}$ and a $1 \mathrm{~km}$ long $2 \mathrm{MGIF}$. The experimental set-up is shown in figure 2. An Agilent laser at $1545.14 \mathrm{~nm}$ is used as the pump source. The pump is fed to the two mode fiber under test through a circulator. For varying input powers, the Brillouin back reflected power is monitored at the third port of the circulator, while measuring the output power at the far end of the test fiber. The launched power into the test fiber is measured using a cut back technique. The $\mathrm{LP}_{11}$ mode is excited in the fiber using a thermally induced fiber based long period grating with a mode purity greater than $20 \mathrm{~dB}$. The SBS threshold (SBST) is calculated from the reflection measurements using the tangent intersection method as shown in figure 3. For the 2MGIF, the SBST are measured as $150 \mathrm{~mW}$ and $462 \mathrm{~mW}$ for the $\mathrm{LP}_{01}$ and $\mathrm{LP}_{11}$ modes respectively, while for $2 \mathrm{MSIF}$, it is measured at a lower value of $33 \mathrm{~mW}$ and $41 \mathrm{~mW}$ respectively. The measured values agrees well with the modeled SBST values as shown in table 2. The higher SBS threshold measured for the $\mathrm{LP}_{11}$ mode accounts for its higher effective area and less overlap with the acoustic modes compared to the fundamental $\mathrm{LP}_{01}$ mode. Going from a step index profile to a graded index profile further changes the acoustic mode profiles resulting in an even lesser overlap between the optic and acoustic modes and a higher SBST.

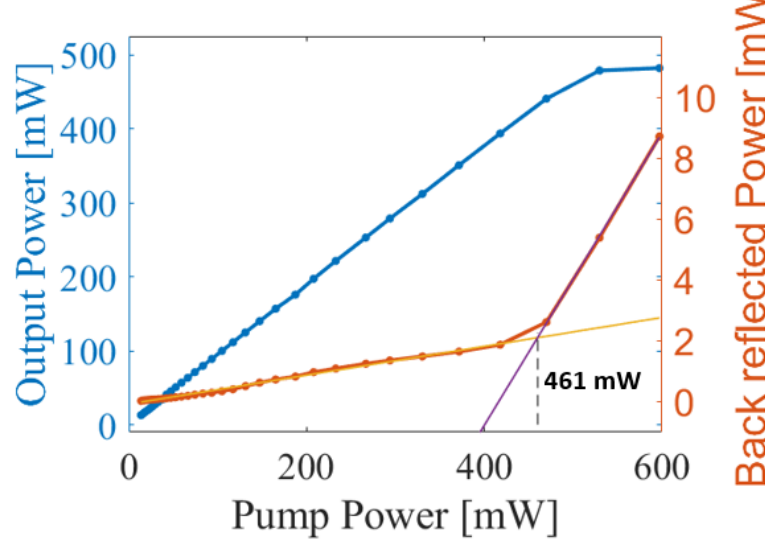

Fig. 3: The measured SBS threshold for the $\mathrm{LP}_{11}$ mode in the $2 \mathrm{MGl}$ fiber

Tab. 2: SBS threshold

\begin{tabular}{|c|c|c|c|c|c|}
\hline \multicolumn{2}{|c|}{} & \multicolumn{2}{c|}{ 2MGIF } & \multicolumn{2}{c|}{ 2MSIF } \\
\hline & & $\mathrm{LP}_{01}$ & $\mathrm{LP}_{11}$ & $\mathrm{LP}_{01}$ & $\mathrm{LP}_{11}$ \\
\hline Length & $\mathrm{km}$ & 1 & 1 & 10 & 10 \\
\hline $\mathrm{P}_{\text {th }}$ mod. & $\mathrm{W}$ & 0.12 & 0.50 & 0,028 & 0.039 \\
\hline $\mathrm{P}_{\text {th }}$ mea. & $\mathrm{W}$ & 0.15 & 0.46 & 0.033 & 0.041 \\
\hline
\end{tabular}

\section{Parametric amplification}

To demonstrate the higher $\mathrm{FOM}_{\mathrm{NL}}$ of higher order mode amplification, we measure the conversion efficiency of intra modal four-wave mixing (FWM) separately for each mode in the 2-mode graded index fiber. 


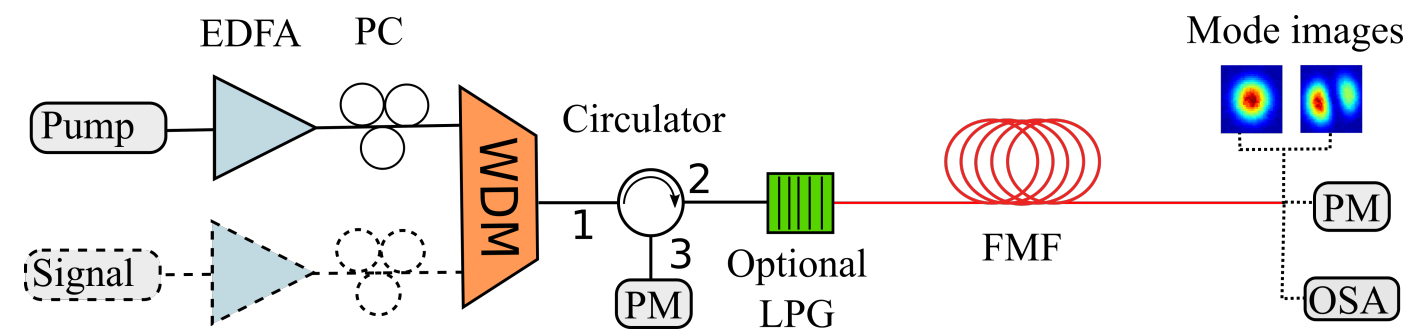

Fig. 2: Setup used in the experiment, dashed lines denote equipment added for FWM experiment. EDFA: Erbium Doped Fiber Amplifier, PC:Polarization controller, PM: Power Meter, LPG: Long Period Grating, FMF: few mode fiber, OSA: Optical spectrum analyzer.

An EDFA amplified tunable laser source is added to our set-up (dashed lines in figure 2), which provides $\approx 60 \mathrm{~mW}$ of optical power. To measure the output spectrum an optical spectrum analyzer is used. The conversion efficiency is given as the ratio between signal and idler power. The modes are launched using either a singlemode to few-mode fiber splice or a broadband long period grating. The pump is kept at 1545.14 $\mathrm{nm}$, and the power is adjusted to the Brillouin threshold for each mode respectively to measure the maximum conversion efficiency. The signal and pump polarizations are aligned by maximizing idler power before each measurement.

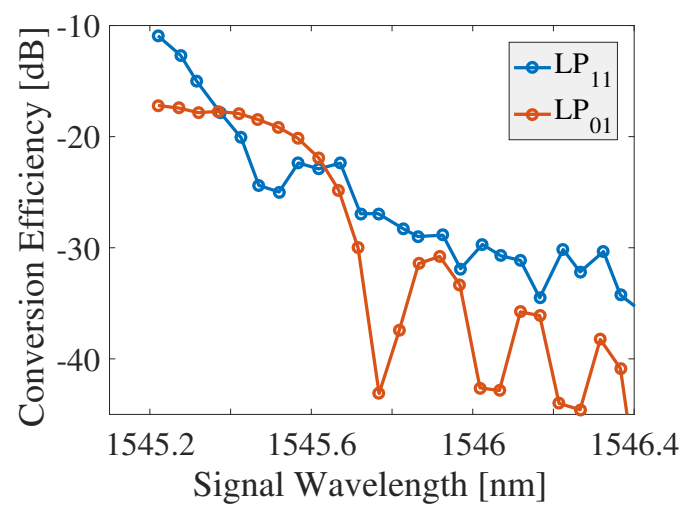

Fig. 4: Conversion efficiency of intramodal four-wave mixing, in a 2-mode graded index fiber.

On figure 4 the conversion efficiency is shown for each mode. Since the dispersion is high in this test fiber, the phase-matching is extremely narrowband. Notice, how the $\mathrm{LP}_{01}$ mode follow the distinctive sinc function pattern, while these are not clearly visible in the $\mathrm{LP}_{11}$ measurement. This could be due to the OSA not perfectly picking up the $\mathrm{LP}_{11}$ mode due to its asymmetric intensity pattern. The maximum conversion efficiency for the $L P_{01} F W M$ is $-17.2 \mathrm{~dB}$, while the $L P_{11}$ mode sees a $6.3 \mathrm{~dB}$ improvement at $-10.92 \mathrm{~dB}$. This corresponds well with the $7.3 \mathrm{~dB}$ improvement, predicted from the measured threshold values. This demonstrates that higher order modes are a viable path for increasing conversion efficiency.

\section{Conclusions}

We show that the non linear figure of merit for SBS limited highly nonlinear fibers $\left(\mathrm{FOM}_{\mathrm{NL}}\right)$ can be improved by optimizing the design of the index profile. Through modeling and experiments it is shown that a graded index fiber profile gives a higher SBS threshold than a step index profile. We have shown a $F O M_{N L}$ of 0.36 using $\mathrm{LP}_{11}$ in an existing fiber with non ideal phase matching. Furthermore, we have shown a theoretical design also with a $\mathrm{FOM}_{\mathrm{NL}}$ of 0.36 with much better dispersion properties yielding an improved phase matching. This is an $80 \%$ improvement compared to the $\mathrm{FOM}_{\mathrm{NL}}$ of 0.21 for conventional single mode $\mathrm{HNLF}^{2}$. Finally, to the best of our knowledge, we demonstrate the first intramodal parametric amplification in a higher order mode.

\section{Acknowledgements}

We thank the Danish research council for financial support through the research grant: DFF -418400433, and OFS Denmark for supplying the fiber.

\section{References}

[1] J. H. Lee et al.,"Experimental comparison of a Kerr nonlinearity figure of merit including the stimulated Brillouin scattering threshold for state-of-the-art nonlinear optical fibers" Optics Letters Vol 30, no. 13 p. 1698 (2005).

[2] L. Grüner-Nielsen et al."Brillouin Suppressed Highly Nonlinear Fibers,"Proc. ECOC, We.1.F.1, Amsterdam (2012).

[3] A. Kobyakov et al., "Stimulated Brillouin scattering in optical fibers," Advances in Optics and Photonics, Vol. 2, no. 1, p. 1 (2010).

[4] L. Grüner-Nielsen et al., "Brillouin Gain Spectra of Few Mode Fibres," Proc. ECOC, P1.SC1.1, Gothenburg (2017).

[5] Cosign deliverable D2.3, pp. 8-10. http ://www.fp7 cosign.eu/wp - content/uploads/2015/09/COSIGNdeliverable $-D 2-3-$ submitted_final_v4.pdf.

[6] E. Nazemosadat et al., "Desing of Highly Nonlinear FewMode Fiber for C-Band Optical Parametric Amplification," Journ. of Lightw. Tech., Vol. 35, no. 14, p. 2810 (2017)

[7] C. Guo et al., "Design of elliptical few-mode fibers for mode-coupling-free parametric amplification," Journ. OSA B, Vol. 35, no. 3, p. 545 (2018). 